\title{
Imaging virus assemblies with in situ cryoEM
}

\author{
Peijun Zhang \\ peijun@strubi.ox.ac.uk
}

Division of Structural Biology, Wellcome Trust Centre for Human Genetics, University of Oxford, Oxford, United Kingdom

Electron Bio-Imaging Centre, Diamond Light Sources, Harwell Science and Innovation Campus,

Didcot, Oxfordshire, United Kingdom

With cryo-electron microscopy, structures of purified proteins and protein complexes can be routinely determined to near-atomic resolution using single particle analysis (cryoEM-SPA) method. Structures of macromolecular assemblies that are intrinsically flexible and dynamic, and often function in higher-order assemblies that are difficult to purify, have recently been analyzed to near near-atomic resolution using cryo-electron tomography and sub-tomogram averaging (cryoEM-STA). The study of these complexes and assemblies in situ using cryoEMSTA, coupled with cryoFIB and correlative and integrative imaging, opens a new frontier in structural cell biology. I will present technology development in cryoFIB and cryoEM-STA, and recent studies of the virus assembly process within native cells to demonstrate the power subtomogram averaging for in situ structure determination. 